UCRL- 98097

PREPRINT

\title{
TIME-DEPENDENT MASS LOSS FROM HOT STARS \\ WITH AND WITHOUT RADIATIVE DRIVING
}

John I. Castor

Stanley P. Owsck i

George B. Rybicki

\section{Trieste Workshop on Pul sation and Mass Loss in Stars}

Trieste, Italy

September 14-18, 1987

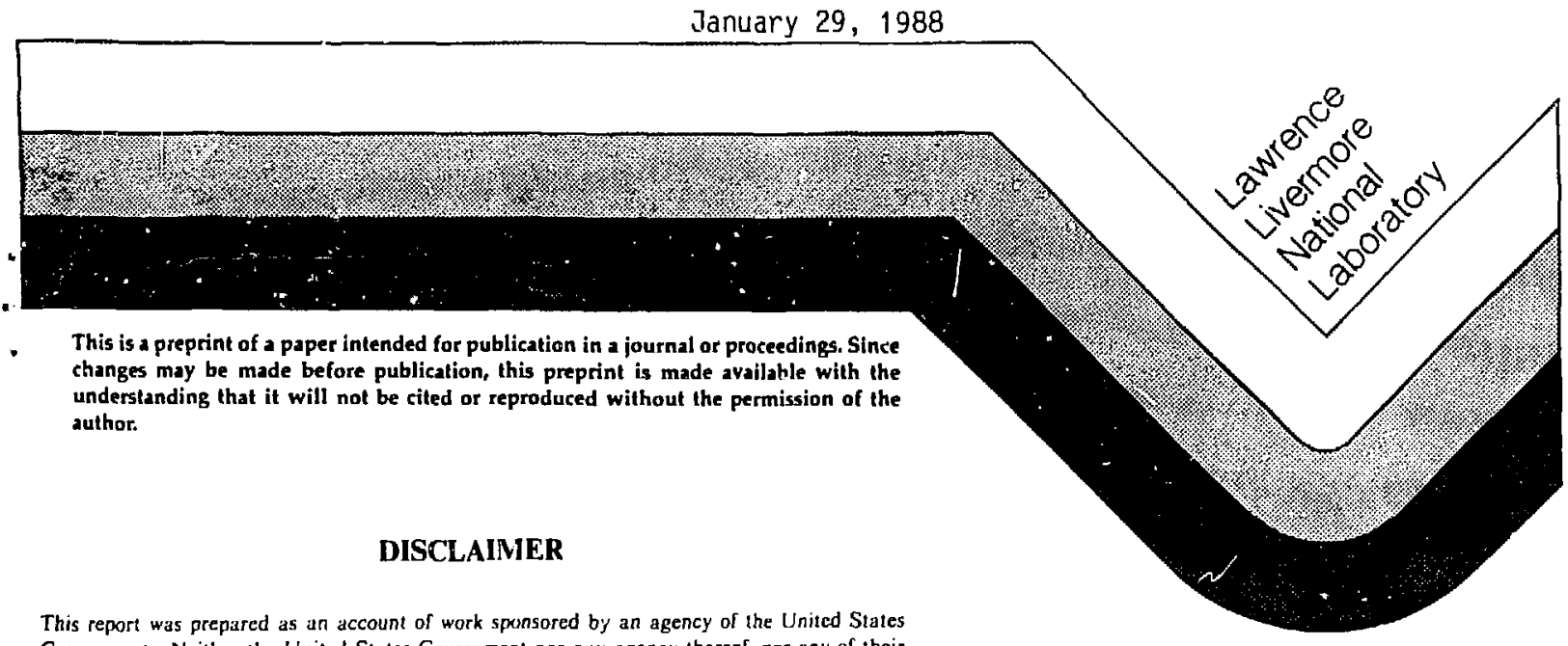

Government. Neither the United States Government nor any agency thereof, nor any of their employees, makes any warranty. express or implied, or assumes any legal liabili:y or resporsibility for the accuracy, completeness, or usclulness of any information, apparatus, product, or process disclosed, or represents that its use would not infringe privately owned rights. Reference herein to any specific commercial product, process, or service by trade name, trademark, manufacturer, or otherwise does not necessarily constitule or imply its endorsem-nt, recommendation, or favoring by the United States Government or any agency thereof. The views and opinions of authors expressed herein do not necessarily state or reflect those of the United States Government or any agency thereof.

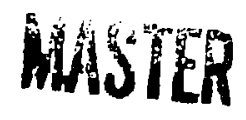

DISTRIBUTION OF THIS UOCUMENI IS UNH.IAHTEO 
UCRL -98097

DE88 008899

\title{
TIME-DEPENDENT MASS LOSS FROM HOT STARS WITH AND WITHOUT RADLATIVE DRIVING
}

\author{
John I. Castor', Stanley P. Owocki², George B. Rybicki ${ }^{3}$ \\ 1 Lawrence Livermore National Laboratory, U. S. A. \\ ${ }^{2}$ Bartol Research Institute, U. S. A. \\ 3 Harvard-Smithsonian Center for Astrophysics, U. S. A.
}

ABSTRACT. A numerical hydrodynamies code is used to investigate two aspects of the winds of hot stars. The first is the question of the instability of the massive radiativelydriven wind of an $\mathbf{O}$ star that is caused by the line shape mechanism: modulation of the radiation force by velocity fluctuations. The evolution of this instability is studied in a model $O$ star wind, and is found, modulo some numerical uncertainty, to lead to wave structures that are compatible with observations of wind instabilities. The other area of investigation is of main-sequence B star winds. Attempts were made to simulate a radiatively-driven and a pulsation-driven wind in a $B$ star, but in each case the wind turned out to be very weak. It is argued that the pulsation-driven wind model is not likely to apply to B stars.

\section{INTRODUCTION}

At the workshop held in April 1986 in Lunteren, The Netherlands, in honor of Professor de Jager at his retirement, several problems involving the dynamical behavior of hot star winds were raised and discussed. At that workshop Baade (1987) described the variations of $O$ and $O f$ stars and how wind fluctuations are tied to their non-radial pulsation. Lamers (1987) discussed the B supergiant variables that have irregular variability and occasionally emit a "puff" of material. The underlying feeling in this discussion was that a radiative instability played a major role in these phenomena, perhapa augmented by a pulsational instability of the stellar envelope. The theory of radiation-driven instabilities was reviewed at the workshop by Rybicki (1987), and the role of stellar pulsation in inducing mass loss was discussed by Castor (1987). A preliminary report on timedependent hydrodynamic calculations of the radiation-driven instability of an $O$ star wiad was given by Owocki, Castor and Rybicki (1987). The present paper is a description of the further progress that has been made toward understanding the dynamics of hot star winds by means of numerical simulations. The first part of our paper is an update of the Owocki, Castor and Rybicki report on $O$-star wind instabilities. We have some interesting results that may shed some light on several wind-instability phenomena. The second part of the paper describes an attempt to assess whether stellar pulsation plays a major role in driving a wind in a $B$ star. The simulations were not very satisfactory in this case, but the answer is a tentative "no". In the remainder of this paper the O-star instabilities are taken up in $\$ \$ 2,3$ and the B-star winds in $\$ 4$. 


\section{INSTABILITY OF RADIATION-DRIVEN WINDS}

There are several observational indicators that the winds from $\mathrm{O}$ and $\mathrm{B}$ stars are hydrodynamically unstable. The three key indicators are (1) the existence of $x$-ray emission that most probably originates in the wind, (2) the time-variability of some of the optical and UV spectral features, and (3) the existence of non-thermal radio emission from the wind.

The $x$-ray emisaion from hot stars, discovered with the Einstein observatory (Harnden et al. [1979]), is most easily understood in terms of emission from regions of hot gas produced by shock waves in the wind. Lucy (1982) proposed a model in which such shock waves are a result of the radiative instability. Cassinelli and Swank (1983) discuss how well this model and others ft the observations, and find some flaws in all; another model of the shocks by Krolik and Raymond (1985) improves the agreement with Cassinelli's and Swank's data. The x-rays also explain the existence (Rogerson and Lamers [1975]) of "superionized" spectral features in the UV: the OVI and NV resonance lines; these can be produced by Auger ionization of the abundant ions.

A number of UV and optical spectral lines are found to show time variations in 0 and $\mathrm{B}$ stars indicative of luctuations in the wind. The $\mathrm{H} \alpha$ line was found by Conti and Frost (1974) to show pronounced night-to-night variations in the O4ef star $\lambda \mathrm{Cep}$, and this phenomenon bas since been found to be fairly general. Many or most of the OB stars have displaced narrow components in the absorption troughs of their UV P Cygni profiles (Lamers, Gathier and Snow [1982]). These are usually present, but sometimes disappear and then are regenerated at a lower velocity within a few hours. This behavior has been studied by Henrichs (1984), and may be due to transitory concentrations ("puffs") of material in the wind.

The third major indicator of wind instability is the existence of non-thermal radio emission from OB star winds, discovered by Abbott, Bieging and Churchwell (1984) and interpreted in terms of a shock acceleration model by White (1995). In this model electrons are accelerated to relativistic energies in the shock fronts, and the synchrotron mechanism produces the emission that is detected. Because the wind is quite opaque in the radio region owing to free-free absorption, the source of the emission needs to be located well out in the wind; the shock model accounts nicely for this.

The linear theory of the instability of a radiatively-driven stellar wind has been reviewed recently by Rybicki (1987) so we will only aummarize the results here. Conflicting results for the stability of a wind driven by the radiation pressure of a large number of apectral lines were found by Abbott (1980), who made use of the Sobolev approximation, and by MacGregor, Hartmann and Raymond (1979), Martens (1979), and Carlberg (1980), who made the optically-thin approximacion. The latter authors all investigated the line-shape instability, which arises from the modulation of the force exerted on the material in the wind, due to absorption and scattering of line radiation, by variation in the fluid's radial component of velocity. Roughly speaking, a positive perturt ation of the velocity produces a positive perturbation of the force, since the parcel of material thus perturbed can absorb or scatter radiation that is less attenuated than it is in the unperturbed state; likewise a negative fluctuation of velocity produces a negative fluctuation of the force. The contradiction between the results was that Abbott did not find instability, but only wave propagation at velocities quite different from the usual sound speed; the other authors found propagation at the sound speed combined with very strong amplification, i.e., instability. Owocki and Rybicki (1984) provided a satisfactory reconciliation of these results by doing a more careful treatment of the radiative transfer for the perturbed system. Abbott's result was found to be correct in the limit that the perturbation wavelength is long compared with the Sobolev length = intrinsic line width in velocity units :- 
velocity gradient; the other result is correct in the limit that the perturbation wavelength is short compared with the Sobolev length. Instability in fact exists at all wavelengths, but is largest for short wavelengths. That there is instability at any wavelength is suffcient to make the wind unstable as a whole. The growth rate at short wavelengths is very large, indeed-aufficient to amplify a disturbance by $e^{100}$ or more as it moves out through the wind.

There is an important fact about the character of the wind instability: it is very probably what is called a drift or convective or advective instability, which means that the growth in time is observed only by riding along with the moving fuid; a fixed observer sees $\mathrm{p}$.turbations that decay in time. In an absolute instability the fixed observer would see growth in time also. This distinction, and the mathematical techniques that may be ayplied to draw it, are discussed by Bers (1983). Owocki and Rybicki (1986) applied these ideas to the radiative instability. The qualitative difference between the types of instability is that an absolute instability is self-exciting, and any perturbation whatever will eventually produce a large amplitude disturbance throughout the gystem-just as in Cepheid pulsation. A drift instability will produce a disturbance in proportion to the perturbation applied. As completely as Owocki and Rybicki have been able to analyze the line-shape instability, it seems to be of the drift type, and therefore its impact on the global structure of the wind depends on what type and magnitude of noise input to the wind exists. Since the amplification is by the enormous factor of $e^{100}$, however, the magnitude of the noise input is of secondary importance compared with the non-linear behavior of the instability.

\section{UNSTABLE O-STAR WINDS}

The Lucy (1982) and Krolik and Raymond (1985) models referred to earlier embody guesses about the global structure that would result from the instability of the radiationdriven wind. Lucy's model assumes that a periodic train of outgoing shocks would result, with each shock separated from the next by such a distance that a critical amount of "shadowing" occurs. The Krolik and Raymond model is, in effect, one of a single isolated shock, and the internal relaxation of the ionization state and gas temperature in the shock front is an important part of the model. Neither the Lucy nor the Krolik and Raymond model is derived from a consideration of the global dynamics of the instability development, but each is instead an intelligent guess of the form the fully-developed instability might take.

Our approach, in the work to be described here, is to simplify the physical model of the gas, and approximate the calculation of the body force due to line radiation pressure, so that it is feasible to make a numerical simulation of the time-dependent stellar wind. The ultimate goal is to discover what kind of structures are created in the wind by the instability, whether of the kind described by Lucy or by Krolik and Raymond, or perhaps of some other kind. The simulation is necessarily crude, so a quantitative comparison with observation will require making a fine-scale calculation based upon the global re-ilts of our coarse simulation.

The numerical techniques we have used are these: an explicit Eulerian hydrodynamics coce that uses operator splitting to perform separate Lagrangian and advection steps; advection using van Leer-type monotonic piecewise-linear interpolation (1977); a standard von Neumann and Richtmyer-type staggered-spatial-mesh Lagrangian step. Hydrodynamics methods similar to ours have been reviewed by Hawley, Smarr and Wilson (1984). For the calculations of the O-star-wind instability the gas temperature was held fixed at a value characteristic of a wind in thermal equilibrium; for reasons of economy 
this temperature was artifically raised by a factor 10 , to a value of $373000 \mathrm{~K}$. The reason for isothermal dynamics is that the post-shock cooling length scale, for massive O-star winds, is generally short compared with other lengths of interest, and apart from a thin relaxation layer the isothermal assumption gives reasonably accurate results. The reason for scaling the temperature is simple: the computer cost varies roughly as temperature to the -1.5 power owing to the need for more spatial zones, a smaller time step, and a finer frequency mesh as the temperature is reduced. The temperature (or sound speed) plays the role of a small parameter in our problem-gravity and the radiative acceleration are the dominant forces through most of the wind-and we believe the large-gcale structure of the wind is insensitive to it. This can be verified after the fact. Scaling the temperature reduces the maximum amplification that the line-shape instability can produce from $e^{100}$ to $e^{30}$. This should still be adequate to drive the instability behavior into the non-linear asymptotic regime.

We have calculated the wind dynamics in two approximations for the radiation force due to lines: one in which the radiation force is calculated using the Sobolev approximation to the radiative transfer (a hypersonic approximation, viz., Castor, Abbott and Klein [1975]) and a second approximation that we call "absorption" in which the force due to the first absorption of the continuum radiation is computed accurately but the force due to radiation emitted or previously scattered within the wind is neglected. In both cases a statistical ensemble of lines with a power-law distribution of strengths is assumed, which enables an analytic summation over the lines. A line-by-line solution for the scattered radiation has so far been too costly to consider in the time-dependent problem. The linear instability theory has shown that the wind is stable if the Sobolev approximation is made, and is definitely unstable in the drift sense in the absorption approximation. It is conceivable that absolute instability may occur if the force due to scattered radiation is taken into account; the necessary analysis to answer this question is not complete.

The boundary conditions applied to the hydrodynamical problem are that the density is fixed at a fiducial value (the nominal photospheric density) at the inner boundary (located at the nominal photospheric radius), while the flow velocity at the inner boundary is free to float. The outer boundary is usually chosen to be at 2-3 photospheric radii, and is such as to permit outgoing waves to be transmitted without reflection. For the initial conditions of the Sobolev-approximation calculations we have taken a crude approximation to the Castor, Abbott and Klein steady wind solution, which agrees with the latter to an accuracy of about 30\%. In some cases this was also used to start absorptionapproximation calculationg, but for other absorption runs either the relaxed Sobolev solution or an ad hoc estimate of the steady absorpticn solution was used.

Figure 1 shows the run of velocity with radius in the Sobolev approximation computed in two ways. One solution was found by running the time-dependent code until a steady state was reached; the other is the singular solution of the ordinary differential equation describing steady low discussed by Castor, Abbott and Klein (1975). We see that the agreement between the two solutions is essentially perfect, given the accuracy of the numerical methods. This indicates that the singular point analysis of Castor, Abbott and Klein is consistent, in this case, with the initial value solution with plausible boundary conditions. The other point demonstrated by this calculation is that in the Sobolev approximation the steady wind is stable, in agreement with the linear theory.

Figure 2 illustrates the rather different situation when the absorption approximation is used. The different curves show the run of velocity at increments of 500 cycles for the first 2500 time cycles. What is apparent is that the perturbation introduced by the departure of the initial condition from the steady solution rapidly grows and creates a strong wave. The further evolution of this problem shows that this wave propagates 


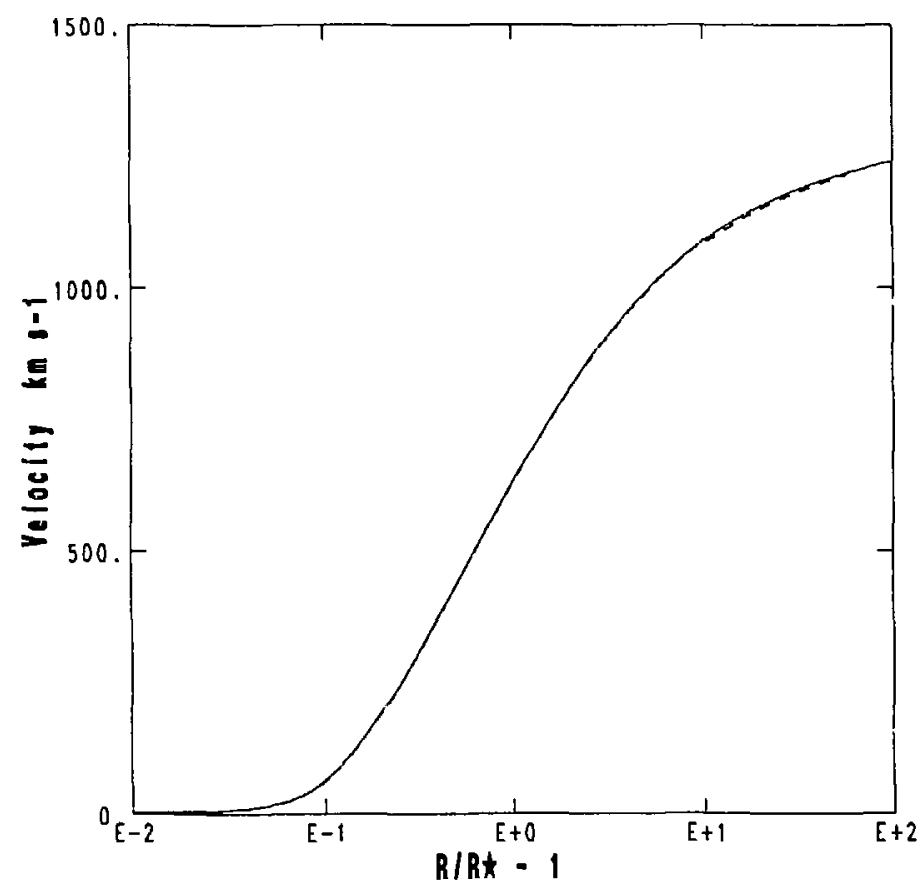

Fig. 1. Velocity distributions for an O-star wind in the Sobolev approximation obtained by a time-dependent calculation (dashed line) and by the steady wind theory (full line).

outward and is succeeded by other waves, until the entire supersonic portion of the wind is affected. The outer region of the wind does not relax to a steady state for as long as the calculation has been continued. This is indicative of the instability of the wind in the absorption appraximation.

The statistical nature of the velocity distribution in the absorption approximation is illustrated in Figure 3. Shown here are the maximum and minimum values of the velocity attained at each radius over a time interval of $4 \times 10^{4} \mathrm{~s}$, as well as the velocity distribution at one typical time. What can be seen is that there is a modulation envelope that has a rapidly increasing amplitude with radius in the region in which most of the acceleration is occurring, but a more nearly constant large amplitude at larger radius where the velocity itself levels off. The typical distribution shows that the modulation consists of waves with a wavelength that is considerable - of order $20 \%$ of the photospheric radius. This oscillation is also quasi-periodic in time, as shown by Figure 4, which displays the time variation of velocity at a certain radius. The quasi-period for this calculation is about $4000 \mathrm{~s}$, which is evidently a natural period of the wind. Test calculations have shown that this period does not vary systematically with the spatial or temporal mesh, although changiug the outer-boundary radius from $2 R$. to $3 R$, significantly increases the 


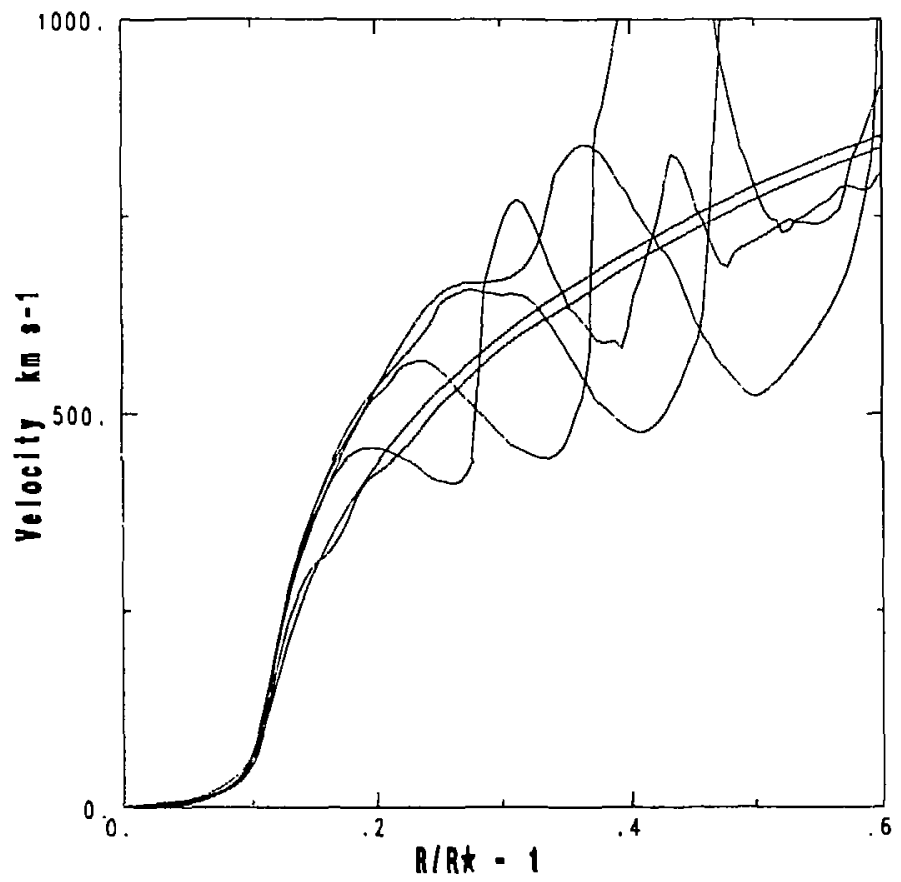

Fig. 2. Run of velocity with radius at intervals of 500 time cycles up to 2500 cycles ( $t=4.9$ hours) for an 0 -star wind calculated in the absorption approximation of the radiation force.

aperiodicity. Reducing the temperature by a factor $\sqrt{10}$ decreased the dominant period by about a factor 2. The run of density, which we have not shown, indicates that the : waves, which originate in the low-velocity region and are amplified as they move outward, are rarefaction waves until a complex wave-interaction region near $r=2 R$, is reached. That is, the spatial variations in density tend to be inverse images of the variations of velocity. The wave velocities that can be inferred are less than the fluid velocity in the waves, indicating that the waves are propagating in toward the photosphere in a frame of reference moving with the fluid. The fluid velocity itself is larger than the relative velocity of wave and fluid, so the wave pattern actually travels away from the center as seen by a fixed observer. The wave velocity and the amplitude and phase of the density variation with respect to the velocity variation are in fact in very good agreement with the linear dispersion analysis of Owocki and Rybicki (1984). The wavelength is rather longer than the Sobolev length, so the wave modes described by $\mathrm{Owocki}$ and Rybicki are approximated by Abbott's (1980) waves, and the mode that we see in our numerical results is the one that propagates inward supersonically relative to the fluid; this is also the mode that, in linear theory, has very rapid outward growth. The growth rate observed numerically is in fair agreement with the linear theory. This is in spite of the fact that the amplitudes are too large for a linear approximation to be justified. 


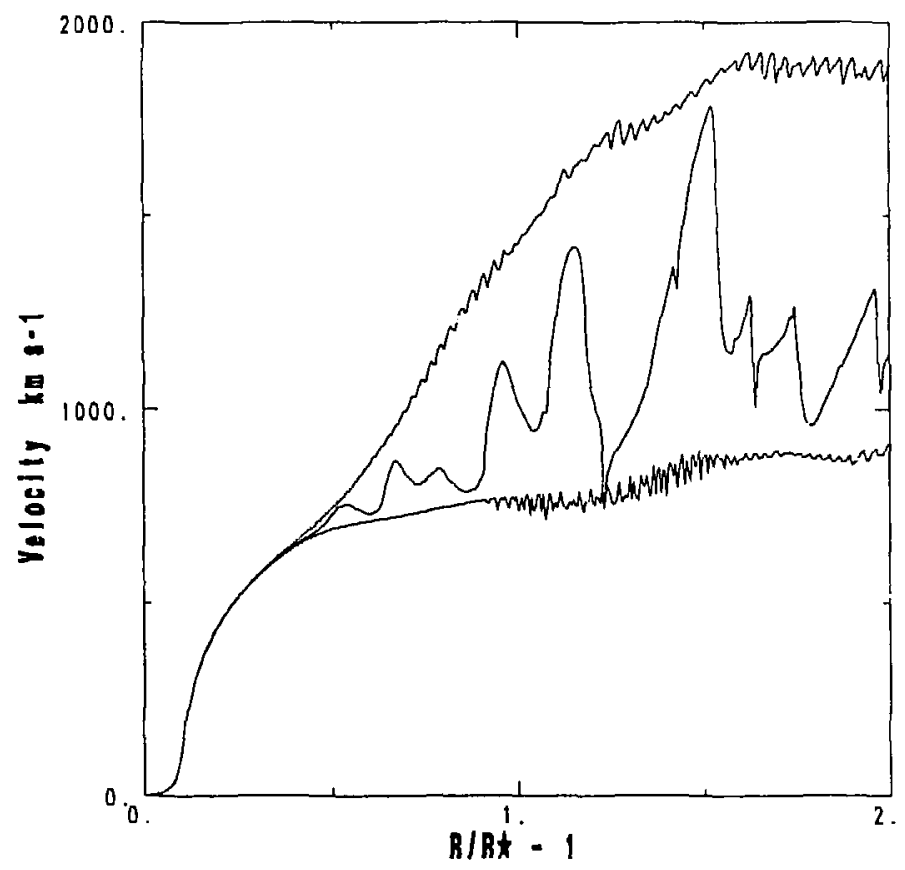

Fig. 3. Velocity us. radius for the 0 -star wind calculation. Shown are the maximum, minimum and fonal velocities taicen over a $4 \times 10^{4} \mathrm{~s}$ time interval at the end of the calculation.

The persistence of the waves in the absorption calculation, without any wave input at the lower boundary, suggests the existence of a global instability of the wind, which corresponds to an absolute instability in the local dispersion analysis; this is puzzling since the linear theory in the absorption approximation suggests that an absolute instability does not exist. We are conscious of the possibility that numerical truncation error could convert a powerful drift instability into a global instability; this, however, should produce a sensitivity to the mesh that we do not observe. Since any real star will have a finite amount of xcoustic noise near the photosphere, the amplification of this source may dominate the self-excitation mechanism for producing waves in the wind. With this in mind we have calculated the response of the wind to an acoustic wave tlux applied at the inner boundary. In place of a fixed lower boundary density and pressure we have allowed the density and presaure to vary sinusoidally in time. For applied density amplitudes around $0.1-1 \%$ and periods of $2000-8000 \mathrm{~s}$ the qualitative behavior of the driven wind ascillations is very similar to the self-excited ones: the same kind of waves appear with similar amplitudes and wavelengths, although now the periodicity is accurate at the forcing period. The extent of the region in which the waves are amplified before reaching saturation decreases with the amplitude of the applied variation. A discussion of all these results may be found in Owocki, Castor and Aybicki (1988). 


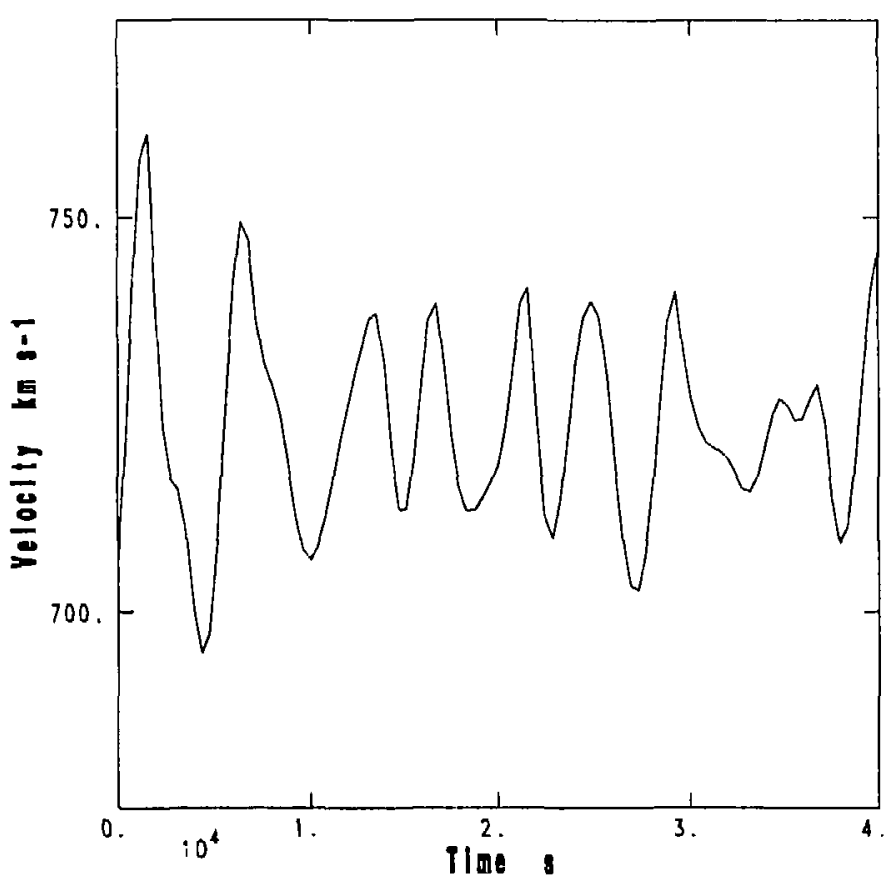

Fig. 4. Velocity vs. time at the fixed radius $1.5 R$. for a portion of the $O$-star wind calculation.

At a radius that is about $2 R$. for the self-excited case the Abbott-type rarefaction waves steepen and form shocks. Specifically, two shocks are generated in the compressed material in the decreasing-velocity part of the waveform, one moving outward into the material at larger radius, and one moving backward through the fluid toward the center; both shocks move to larger radius as viewed in a fixed frame. The forward and reverse shocks begin close to each other and are separated by a very dense layer at an intermediate velocity. As this whole structure moves to larger radius the dense layer decompresses and the forward and reverse shocks separate. Soon the forward shock of one pair collides with the reverse shock of another pair, and in this interaction the velocity-density relation changes yet again. In the region exterior to this point the shock interactions become very complex and the strengths gradually diminish with radius. The summary of where shock waves are found in the wind, and at what strengths, is that the shocks are seen first at an appreciable height above the photosphere (at $r \approx 2 R$, in our self-excited model) and are essentially at maximum strength at that point; the strength decrtases with radius beyond there.

There are a nuraber of interesting aspects of the external appeasace of the wind as modified by the presence of the strong waves and shocks. The shocks will produce non-thermal radio emission and $x$-ray emigsion, and the latter will in tun produce superionization, as in the models of Lucy (1982) and White (1985). Quantitative agreement 
of the $x$-ray emission with observation requires shock jumps of order $600 \mathrm{~km} \mathrm{~s}^{-1}$ and that each parcel of wind material be shocked approximately once as it lows away from the star. These figures are in reasonable agreement with the kinematics of our model. The concentration of the shock emission away from the base of the wind, where a large part of the total wind column density occurs, ameliorates the problem of the observed lack of a dip in the $\mathrm{x}$-ray spectrum at the CNO K edges. A consequence of the density inhomogeneity is that the wind has an appreciable clumping factor, defined as $\left\langle\rho^{2}\right\rangle /\langle\rho\rangle^{2}$, where the averages are taken over time at a given radius. An estimate for one of our models is that a clumping factor of about 7 exists in much of the high-velocity region. This causes the optically-thin free-free emisgion and the emission in the far wings of $\mathrm{H \alpha}$ to be greater than in a non-clumpy wind with the same $\dot{M}$.

Perhaps the most interesting observable aspect of the waves is the consequence for P-Cygni profiles. Because the density luctuations are strongly correlated with the veloeity fluctuations in each region of radius (even though the sense of the correlation varies), the column density of the wind is quite non-uniformly distributed with respect to velocity. There is a preference for a velocity close to $v_{\min }$ for the material out to the radius where the shocks form; there is also a preference for the velocity intermediate be. tween $v_{\max }$ and $v_{\operatorname{maln}}$ of the compressed layer between the shocks of the forward-reverse shock pair. These preferred velocities are insensitive to the exact phase of the various waves, and therefore they are also preferred velocities when an ensemble average is taken of the column density distribution with respect to velocity. Figure 5 shows a set of timeaveraged absorption profiles (the blue side of P-Cygni profiles if the emission were entirely absent) for lines of various strengths. These profiles have relatively narrow bumps not unlike the displaced narrow components observed in the UV resonance lines of most $O B$ stara. A question we cannot address at present concerns the visibility of any variations in the narrow crmponents; a short horizontal coherence length of the waves would argue against seeing ar: $y$ such variation.

Although we are several steps away ïrom making a careful quantitative comparison between our hydrodynamic models and observations of $\mathrm{OB}$ stars, we are encouraged by how well the kinematics we obtain fits with the current empirical models.

\section{B-STAR WIND GALCULATIONS}

Because near-main-sequence early $B$ stars are near the threshhold luminosity for a radiation-driven wind (Abbott [1979]), and because most or all of these stars are thought to be non-radial pulsators (See Baade [1988] and references therein.), it is important to consider whether mass loss can be produced in these stars by the pulsation with or without the assistance of radiation driving. In this section we address this question using the hydrodynamic code that was developed to study the O-star instabilities. The work that will be described is very preliminary, and represents only a first attack on the subject; as will be seen, further work must correct some of the approximations made here and overcome some obst iles that were encountered.

The general uleas about mass loss that is caused by shock waves associated with stellar pulsation have been discussed by Castor (1987). In essence, the mechanism is that the pulsation, which may be nearly sinusoidal near the photosphere, suffers distortion of the wave form at higher levels of the atmosphere-steepening-and a shock wave is produced at a certain level that depends on the initial wave form and the period as well ag the properties of the atmosphere. The shock wave strengthens in a definite way with increasing height until it reaches a limiting strength-height relation for which the periodic 


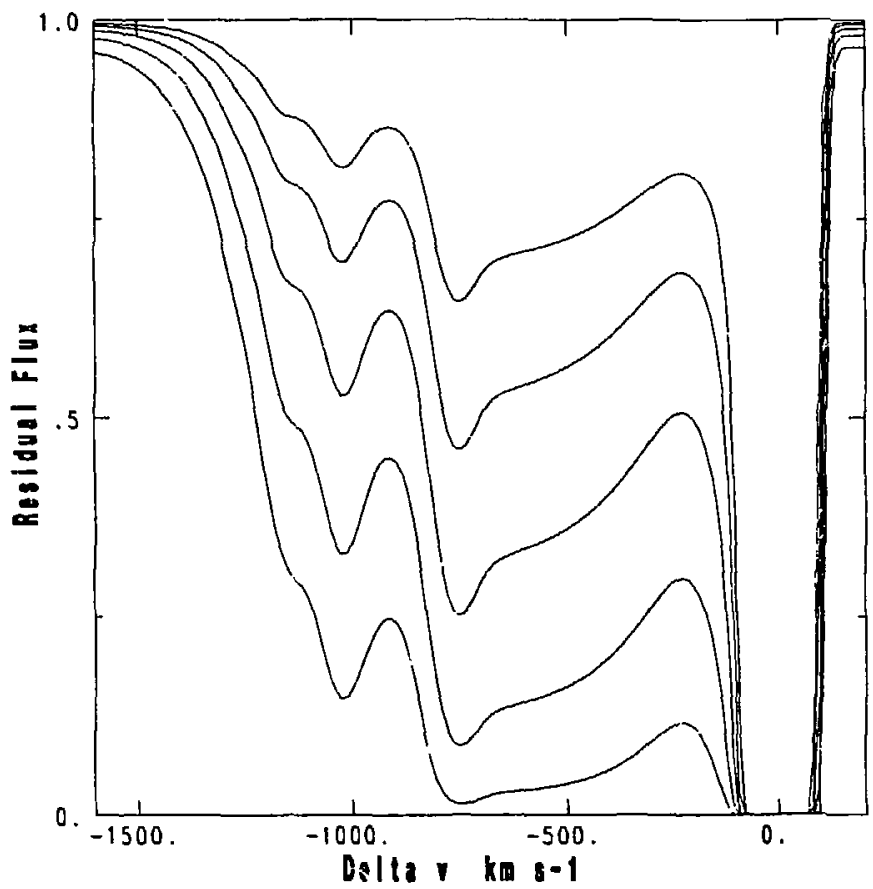

Fig. 5. Ensemble-average emergent line profiles, omitting emission. The ordinate is the residual flux in continuum units; the abscissa is the wavelength displacement from line center in velocity units $\left(\mathrm{km} \mathrm{s}^{-1}\right)$. The different curves show the profile for different line opacities in a geometric sequence with the ratio $10^{1 / 4}$.

shock impulse is able to support the atmosphere against gravity with only a negligible contribution from pressure between shock passages. If this condition is reached while the post-shock gas, by virtue of being sufficiently dense, is able to cool to ambient temperature within a small fraction of the period, then the shock waves produce only support of the atmosphere, not mass loss. At sufficient height in the atmosphere, however, the cooling becomes ineffective, and the gas that is beated by the passage of a shock remaing hot. This creates a corona, which terminates in a pressure-driven wind of the Parker type. The mass loss rate in the wind scales with the base pressure of the corona, which is determined by the density dependence of the post-shock cooling rate.

A numerical illustration of shock-driven mass losg for a $B$ star with $M=15 M_{\odot}$ and $R=6.4 R_{\odot}$, pulsating with a period of either 3 hours or 12 hours, indicates that the shock first forms in a layer where the density is already quite low, and the cooling becomes ineffective (the shock changes from isothermal to adiabatic) while the shock is still increasing in strength to the limiting value. The Parker-type mass loss rate can be estimated to be of order $10^{-12} \mathrm{M}_{\odot} \mathrm{y}^{-1}$ for the 3 hour period, and $10^{-11}$ for the 12 hour period. The low values are due to a combination of two factors. First, both 3 hours and 
12 hours are long compared with the atmosphere's natural period $4 \pi a / g$, which is 0.59 hours. (This is the longest period of a sound wave for propagation in the atmosphere, rather than evanescence; it is also the period with which the atmosphere rings following an impulse.) This makes a shock difficult to form-the pulsation steepens into a shock only bigh in the atmosphere. The second factor is the excellent cooling efficiency of the shocked gas in an OB star atmosphere. This makes the base pressure of the corona very low. The low estimates of shock-driven mass loss would seem to rule out this mechanism as a major player in early B star winds, whose observed rates of mass loss are of order $10^{-10}$ or more, unless the driving pulgation has components with shorter periods or the radiation force has a compounding effect on the pulsation.

We have tried to use our hydrodynamic code to corroborate numerically this assessment. The model we have calculated has the mass, $15 M_{\odot}$, and radius, $6.4 R_{\odot}$, mentioned above, and a luminosity of $10^{4} L_{\odot}$, so the effective temperature is $23000 \mathrm{~K}$ and the surface gravity is $10^{4}$. We assume that the radiative equilibrium wind temperature is the scaledup value $4 \times 10^{5} \mathrm{~K}$; the scaled sound speed is $82 \mathrm{~km} \mathrm{~s}^{-1}$, about 5 times higher than a more realistic value $17 \mathrm{kms}^{-1}$. For these models we have used an energy equation including a crude optically-thin cooling law (cooling per unit volume proportional to $\rho^{2} / T_{e}^{1 / 2}$ ), and a corresponding heating rate to ensure radiative equilibrium at $4 \times 10^{5} \mathrm{~K}$. Some of the model calculations include stellar pulsation (as a time-dependent inner-boundary pressure) with a period of 60000s (16.7 hours) and an amplitude consistent with an Eulerian logarithmic pressure variation at the boundary of 1 . The velocity variation at the inner boundary turng out to be $7.5 \mathrm{~km} \mathrm{~s}^{-1}$, in very good agreement with the usual standing-wave surface boundary condition for pulsation. The period chosen is a compromise between the observed range 3-12 hours and the range 14-56 hours that would keep the period in the correct ratio to the scaled resonance period $4 \pi a / g=2.8$ hours.

For the first calculation we use a constant-pressure boundary condition and include the radiation force in the absorption approximation. A techrical point about the power-law ensemble of driving lines becomes important hers. It is that the atatistical distribution of line opacities must include an up: er cut-off, since otherwise the distribution contains an infinite total oscillator strength, which means that an infinite total force can be exerted on the matter when all the lines are optically thin; this limit is approached when the velocity gradient becomes very large. To avoid this unph, rical result, and to ameliorate the numerical difficulties that arise with large velocity gradients, we set this cut-sff at a moderate value related to the steepest gradient our radial mesh can support. We find that if the cut-off appropriate for $O$ atars is used for the B-star model then the star can not have a radiatively-driven wind-there would not be any optically-thick lines, a necessity for the GAK theory. This is related to Abbott's (1979) lower limit in luminosity for self-excited winds. In order to operate with the hypothesis that our B star does have a radiatively-driven wind, we have increased the value of the upper opacity cut-off by an amount suficient to allow a Sobolev-type wind to exist. The valus of $\dot{M}$ for the CAK wind of our $B$ star is $1.8 \times 10^{-8} \mathrm{M}_{\odot} \mathrm{y}^{-1}$ (with no upper opacity cut-off at all), and with the modified value of the cut-off it is $4.6 \times 10^{-9}$. The outer boundary for this calculation was set at $54 R$.

The time-dependent hydrodynamic calculations with the absorption approximation are initialized with the approximate Sobolev steady-flow solution. This turus out to be a rather poor approximation to the steady solution in the absorption approximation, and about 100,000 time cycles of calculation, representing 250 hours of star time, are required to approach a statistical steady state. (For comparison, the How time through the region from $r \approx 1.5 R$, to $r \approx 2.5 R$, is about 1.5 hours.) Even at this point the flow is not very steady. The run of yelocity with radius shown in Figure 6 is typical at this phase: there 


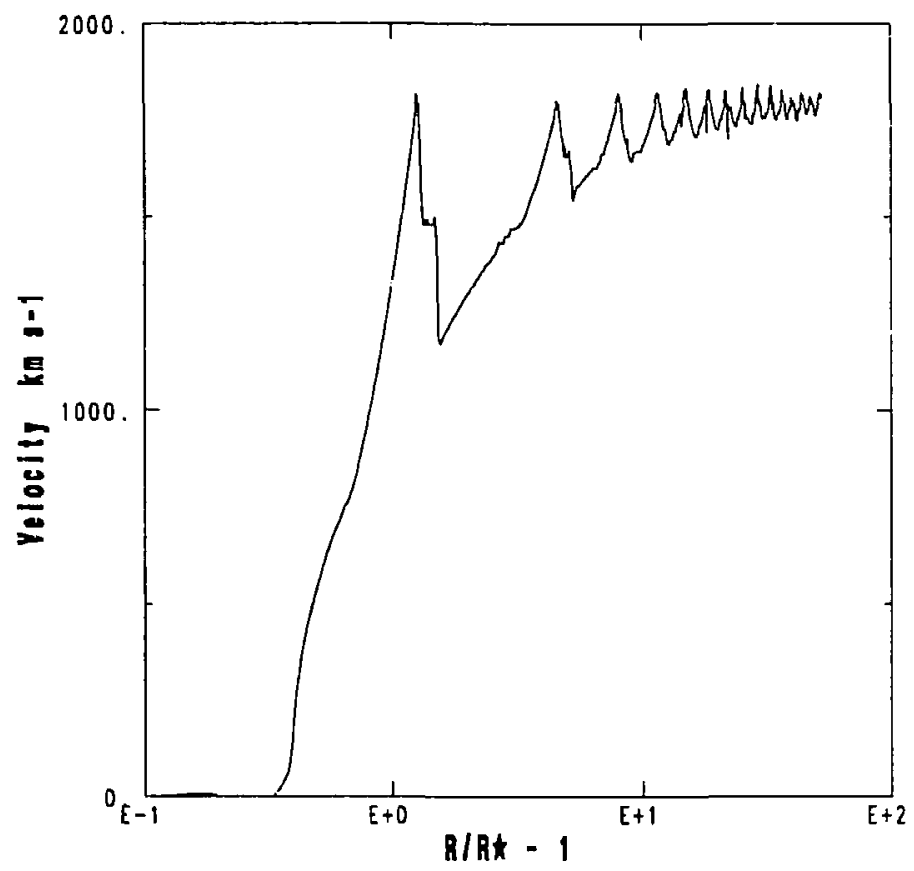

Fig. 6. Velocity us. radius at the end of the calculation of the B-star wind with the radiation force but without pulsation.

is a familiar-looking rising curve on which are superimposed shock waves that decrease in strength with increasing radius; the first shock, near $r \approx 1.6 R_{*}$, is very strong indeed. The waves are quite regularly spaced in time at an interval of 2.5 hours, the game as the ringing period of the atmosphere. Thus these waves should be thought of as part of the transient behavior of the wind as it adjusts to a steady state. Indeed, the wave intensity shows a slow decrease over the duration of the calculation.

The mass density of the wind greatly decreased during the calculation; much of the mass originally present in the wind was accreted by the star, and another large amount was lost at the outer boundary without being replaced from below. Figure 7 shows a comparison of the initial run of density with the average run of density obtained near the end of the calculation. The density in the wind has evidently dropped by a factor of more than $10^{3}$. The mass flux is conserved in an intermediate-radius portion of the wind, as shown in Figure 8. The value of $\dot{M}$ suggested here, $3.3 \times 10^{-12}$, is 1000 times less than the steady Sobolev value and almost $10^{4}$ times less than the simple CAK formula. The reason it is so much lower is very likely that the absorption approximation gives a much lower force in the sub-sonic region than does the Sobolev approximation; the absorption approximation omits the contribution of scattered radiation, which is very important in the sub-sonic region, especially since we assume that the photospheric spectrum has 


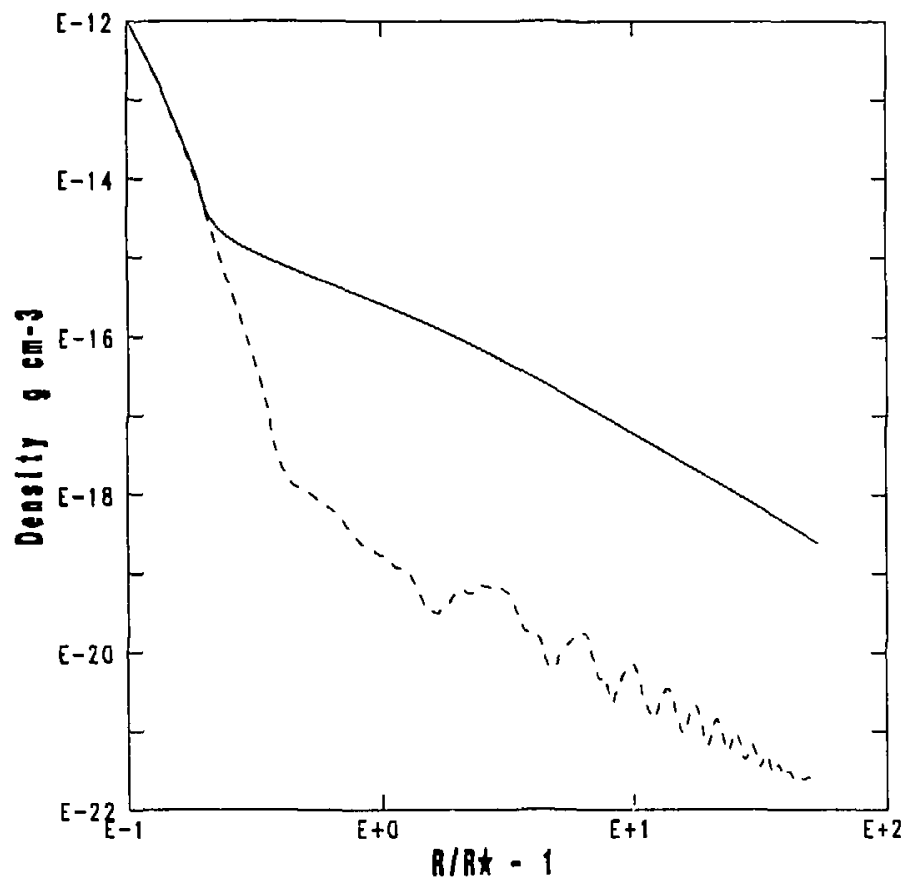

Fig. 7. Initial distribution of density (full line) and time-average final distribution (dashed line) for the B-star wind with the radiation force but without pulsation.

rather black absorption features at the positions of the driving lines. This has a moderate effect on the mass loss and velocity distribution for the $\mathrm{O}$-star models, but a dramatic effect on the B-star model. We are forced to conclude that we cannot calculate a very realistic model of a radiatively-driven B-star wind.

For our other B-star calculation we omit the radiation force and calculate the wind of a pulsating B star; this is a straight gas dynamics calculation including the crude heating-cooling model described above. The boundary condition was the oscillating pressure also described earlier. For the initial state we took an ad hoc model constructed by making a Parker wind, which was very close to hydrostatic equilibrium in the region we calculated, then adding a density "halo" with a $r^{-2}$ distribution; the maguitude of the latter contribution was based on a guess of the eventual pulsation-driven mass loss. This calculation, like the preceding one, is very slow to relax. The results shown here were obtained after more than 600,000 time cycles, equivaleat to 98 pulsation periods. Again, the relaxation is incomplete. Figure 9 shows the maximum, minimum and average velucity versus radius for one pulsation period at the end of the run. Figure 10 shows the corresponding density distributions, and Figure 11 shows the mean temperature distribution. Also shown in Figure 11 is the "Parker temperature", defined as the temperature for which the sonic point of an isothermal Parker wind could occur at each 


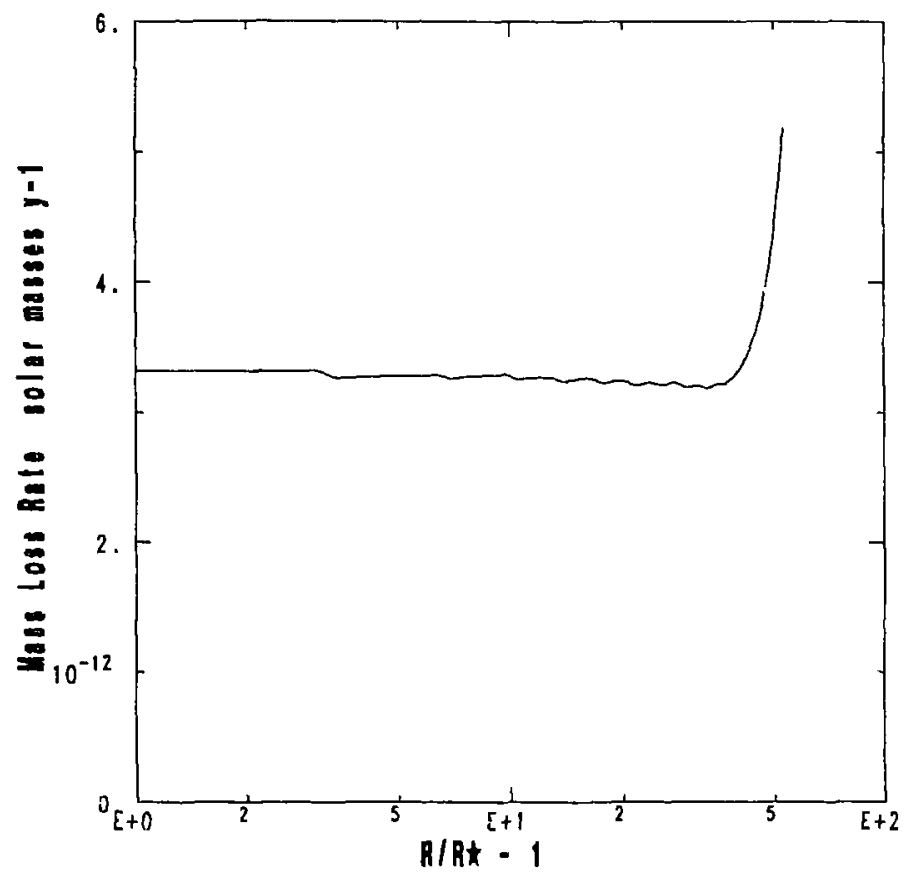

Fig. 8. The time-average rate of mass flow through each radius vs. radius obtained near the end of the calculation of the B-star wind with radiation force but without pulsation.

given radije. What "ve discover by examining these figures is that shocks do not form in this calculation below the height at which thr: cooling becomes ineffective, $r \approx 1.4 R$, where the iemperature jumps abruptly to a coronal value: $4 \times 10^{6} \mathrm{~K}$. In the hot region above there are shocks, but they are weak, and serve only to maintain the temperature of this region. The velocity amplitude is everywhere subsonic and smaller than the escape velocity. Interestingly, the temperature closely approaches the Parker temperature in the neighborhood of 5-10 $R_{\text {. }} ; v_{\max }$ attains a maximum near $r=5 R_{\text {. }}$ at a sub-sonic value in a way reminiscent of the sub-critical Parker solutions.

The reason why shocks do not form below the coronal transition in this model must be that the velocity amplitude at the lower boundary, $7.5 \mathrm{~km} \mathrm{~s}^{-1}$, is only Mach 0.1 , rather than Mach 1 as assumed in the earlier estimate and as suggested by the observations. This is a consequence of using the scaied-up radiative equilibrium temperature. Although this calculation is obviously not at all relaxed, the order of magnitude of the net mass flow observed in the range $r \approx 10-20 R$, is a few $\times 10^{-15} M_{\odot} y^{-1}$. The mass loss rate of the steady pulsation-driven wind is not likely to be larger than this. Fortuitously, this agrees with the order of magnitude estimated by Castor (1987) for mass loss driven by pulsation with a period of 12 hours. The agreement must be considered fortuitous since the estimate was predicated on the existence of strong ahocks, while our calculation 


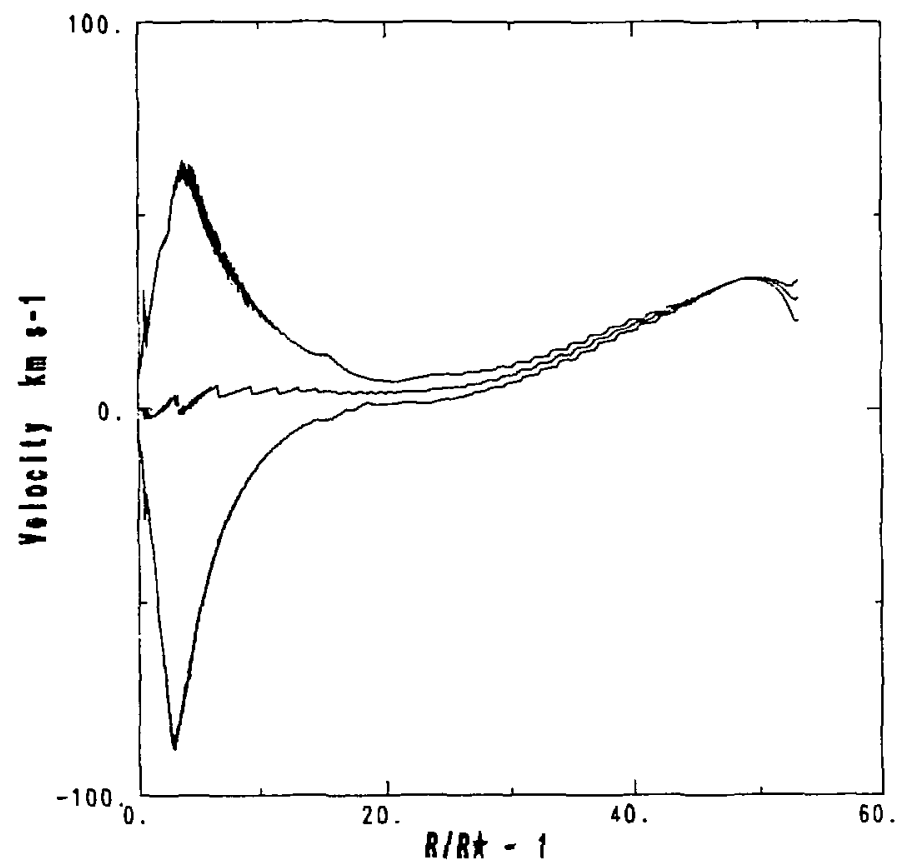

Fig. 9. Maximum, minimum and mass-weighted average velocity us. radius for the pulsating B-star calculation.

produced an extended cornna instearl.

\section{SUMMARY}

We have used a time-dependent Eulerian hydradynamics code to investigate some problems involving winds of hot stars. One such problem is the nature of the largeamplitude disturbances that result in the wind of an $O$ star from the line-shape instability of a radiatively-driven wind. Calculating the hydrodynamics in the absorption approximation-neglecting line emission in the wind-verifies the existence of the instability, and the further evolution leads to interesting medium-scale wave structures that ? 7 ee in several respects with the requirements of current empirical models of 0 star wiilds. The question remains open whether these waves are truly self-excited or are driven by some, possibly very small, noise input from below the photosphere; the wind properties are expected to be quite similar in either case.

In order to elucidate the mass loss mechanism in near-main-sequence early B stars we have calculated models with only radiation driving, and with pulsation driving instead. Both models are less than satisfactory from a technical point of view, but in each case a mass-loss rate that is orders of magnitude less than that observed for some B stars 


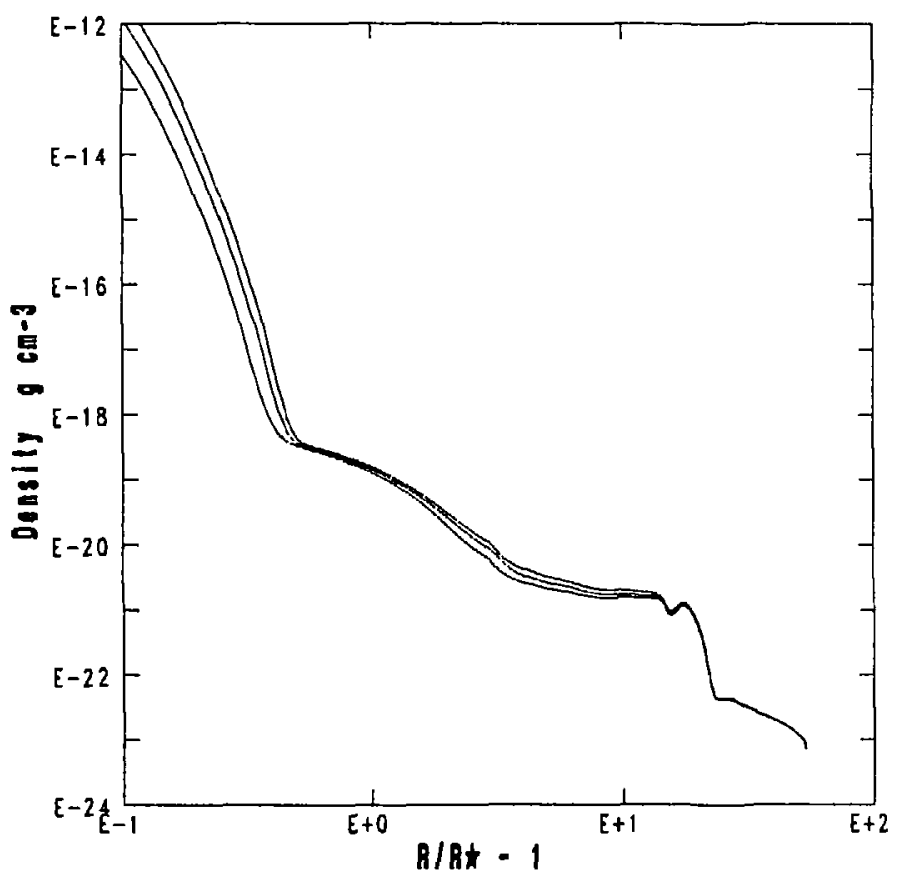

Fig. 10. Maximum, minimum and average runs of density with radius for the pulsating B-star calculation.

is indicated. The prospects for a successful pulsation-driven wind model in these stars look poor. A radiatively-driven wind model for the $\mathrm{B}$ stars might succeed, but only by relaxing some of our approxinations, in particular the absorption approximation and the use of the power-law line opacity diatribution with an ad hoc cut-off.

This work was performed in part under the auspices of the U. S. Department of Energy by the Lawrence Livermore National Laboratory under Contract No. W-7405ENG-48, and was supported by an Institutional Research and Development grant from L.L.N.L. S. P. O. also acknowledges support from NSF grant AST 86-11824 and NASA grant NAG-8-613. 


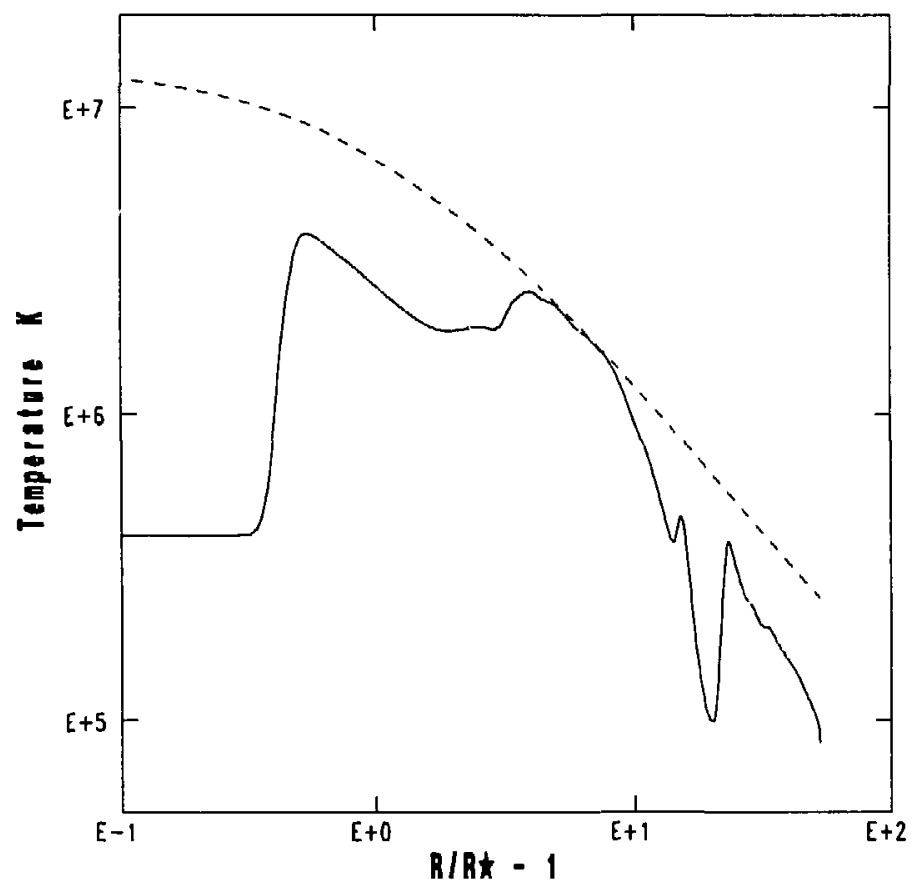

Fig. 11. Full line: mass-weighted average temperature v8. radius for the pulsating Bstar model. Dashed line: Parker temperature $G M / 2 R r$. 


\section{REFERENCES}

Abbott, D.C. 1979, in Mass Lass and Evolution of $O$ Type Stars, ede. P. Conti and C. de Loore (Dordrecht: Zuidel), p. 237. . 1980, Astrophys.J., 242, 1183.

Abbott, D.C., Bieging, J.H. and Churchwell, E. 1984, Astrophys.J., $280,671$.

Baade, D. 1987, in Instabilities in Luminous Early Type Stars, eds. H.J.G.L.M. Lamers and C.W.H. de Loore (Dordrecht: Reidel), p. 73. . 1988, in $O$, Of and Wolf-Rayet Stars, eds. P.S. Conti and A.B. Underhill, NASA/CNRS Series on Nonthermal Phenomena in Stellar Atmospheres, in press.

Bers, A. 1983, in Handbook of Plasma Physics, Volume 1: Basic Plasma Physics I, eds. A.A. Galeev -nd R.N. Sudan (Amsterdam: North-Holland Publishing Company), p. 451.

Carlberg, R.G. 1980, Astrophys.J., 241, 1131.

Cassinelli, J.P. and Swank, J.H. 1983, Astrophys.J., $271,681$.

Castor J.I. 1987,in Instabilities in Luminous . Early Type Stars, eds. H.J.G.L.M. Lamers and C.W.H. de Loore (Dordrecht: Reidel), p. 159.

Castor, J.I., Abbott, D.C. and Klein, R.I. 1975 Astrophys.J., 195, 157.

Conti, P.S. and Frost, S. 1974, Astrophys.J., 190, L137.

Harnden, F.R., Jr., Branduardi, G., Elvis, M., Gorenstein, P., Grindlay, J., Pye, J.P., Rosner, R., Topka, K. and Vaiana, G. S. 1979, Astrophys.J., 234, L55.

Hawley, J.F., Smarr, L.L. and Wilson, J.R. 1984, Astrophys.J.(Suppl.), 55, 211.

Henrichs, H. 1984, in Proc. 4th European IUE Conference, ESA SP-218.

Krolik, J. and Raymond, J.C. 1985, Astrophys.J., 298, 660.

Lamers, H.J.G.L.M. 1987 in Instabilities in Luminous Early Type Stars, eds. H.J.G.L.M. Lamers and C.W.H. de Loore (Dordrecht: Reidel), p. 99.

Lamers, H.J.G.L.M., Gathier, R. and Snow, T.P. 1982, Astron.Astrophys., 118, 245.

Lucy, L.B. 1982, Astrophys.J., 255, 286.

MacGregor, K.B., Hartmann, L. and Raymond, J.C. 1979, Astrophys.J., 2ذ1, 514.

Martens, P. 1979, Astron.Astrophys., 75, L7.

Owocki, S.P., Castor, J.I. and Rybicki, G.B. 1987, in Instabilities in Luminous Early Type Stars, eds. H.J.G.L.M. Lamers and C.W.R. de Loore (Dordrecht: Reidel), p. 269. .1988, in preparation.

Owocki, S.P. and Rybicki, G.B. 1984 Astrophys.J., $284,337$. . 1986, Astrophys.J., 309, 127.

Rogerson, J. and Lamers, H.J.G.L.M. 1975, Nature, 256, 190.

Rybicki, G.B. 1987, in Instabilities in Luminous Early Type Stans, eds. H.J.G.L.M.

Lamers and C.W.H. de Loore (Dordrecht: Reidel), p. 175.

van Leer, B. 1977, J.Comp.Phys, 23, 276.

White, R.L. 1985, Astrophys.J., 289, 698. 